\title{
BILATERAL CONTINUOUS ERECTOR SPINAE PLANE BLOCK GLIDING UP RESPIRATORY FUNCTION IN A POLYTRAUMA CASE
}

\author{
R. Santos Pinheiro, A. Vasconcelos Pereira, B. Aguiar Tavares da Silva, L. Rego, N. Costa \\ Vila Franca de Xira Hospital, Anesthesiology, Vila Franca de Xira, Portugal
}

\section{BACKGROUND}

Rib fractures, lung contusion and atelectasis are common in polytraumatized patients and require effective analgesia to prevent respiratory complications. Managing pain may be challenging, usually requiring invasive procedures. Erector Spinae Plane Block (ESPB) is a recent regional technique of choice in patients with multiple rib fractures due to its safety and simplicity.

\section{CASE-REPORT}

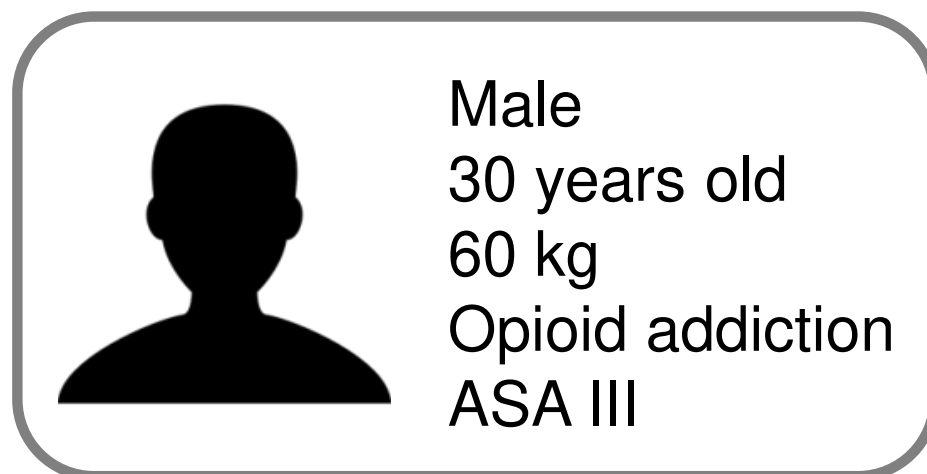

At D6 post admission

Fever

Tachypnea

Purulent sputum

Inadequate pain relief (NRS 7 - at rest)
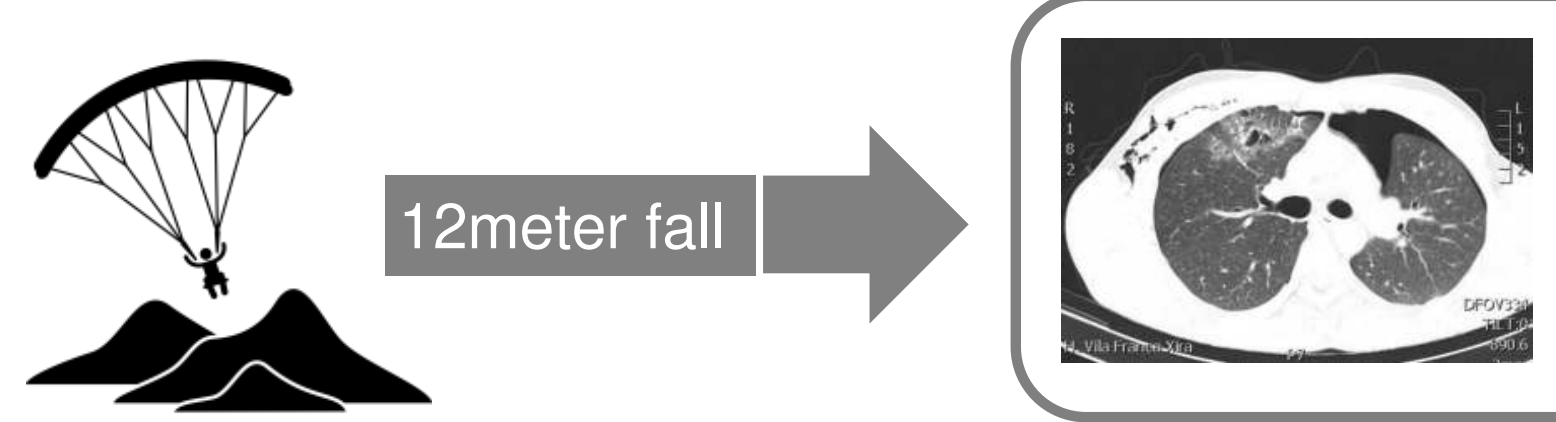

Rib fractures

Sternum fractures

Bilateral pneumothorax

Lung contusion

Intermediate Care Unit admission

\section{METHODS}

\section{NEUROAXIS BLOCK \\ Thoracic Epidural}

Relative $\mathrm{Cl}$
PERINEURAL BLOCK

\section{Continuous Bilateral ESPB}

\section{RESULTS}

Opioid-free pain relief (NRS 0 - at rest / 3 - with mobilization) and ventilation improvement (eupneic, $\mathrm{pH} 7,42 ; \mathrm{pO}_{2}$ $87 \mathrm{mmHg} ; \mathrm{pCO}_{2} 40 \mathrm{mmHg} ; \mathrm{SpO}_{2} 99 \%$ with $0,21 \mathrm{FiO}_{2}$ ) were achieved.

We also noticed a reduction in the inflammatory parameters.

\section{DISCUSSION}

Thoracic trauma is related with respiratory complications (pneumonia, atelectasis, respiratory failure...) that can be reduced by efective analgesia. Neuroaxis or perineural blocks are commonly used analgesic techniques.

We choosed to perform a continuous bilateral ESPB instead of approaching neuraxis, as it is a safer and an appealing alternative considering his current respiratory infection. We not only managed to do an opioid-free analgesia in a patient with opioid addiction, but also improved respiratory function as measured by the ABG analysis optimization.

ESP block improves ventilatory dynamics and optimizes respiratory complications, while being an effective analgesia for patients with thoracic trauma. 